Supplemental material

\title{
Iron (III) silicotungstate: an efficient and recyclable catalyst to converting glycerol to solketal
}

\author{
Marcio Jose da Silva, Alana alves rodrigues and Milena Galdino TeiXeira* \\ Chemistry Departament, Federal University of Viçosa, Viçosa, Minas Gerais State, Brazil, \\ zip code: $36570-000$ \\ silvamj2003@ufv.br
}

\section{List of Figures}

Figure 1SM. EDS spectra and MEV images of the silicotungstic acid and their metal exchanged salts

\section{List of Tables}

Table 1SM. Crystallite size of of silicotungstic acid and their transition metal salts

Table 2SM. Hydration level of the silicotungstic acid and their transition metal salts

Table 3SM. Surface, volume, and pores size of silicotungstic catalysts 


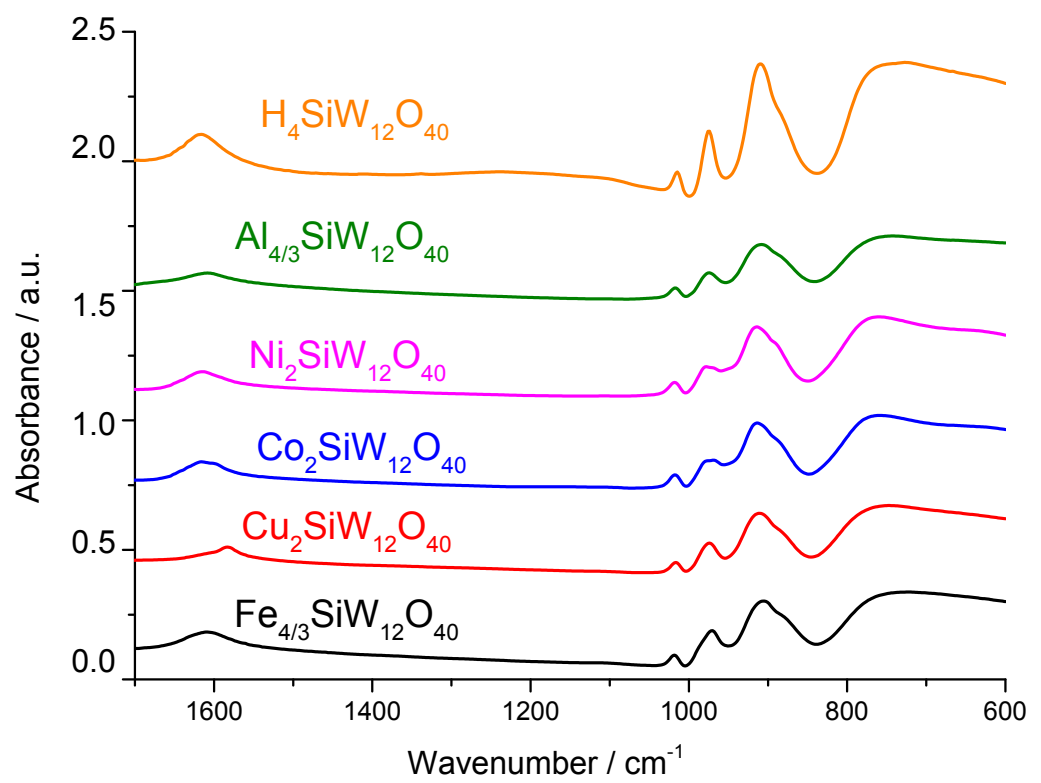

Figure 1SM. FT-IR/ ATR spectra of the silicotungstic acid and their metal exchanged salts 

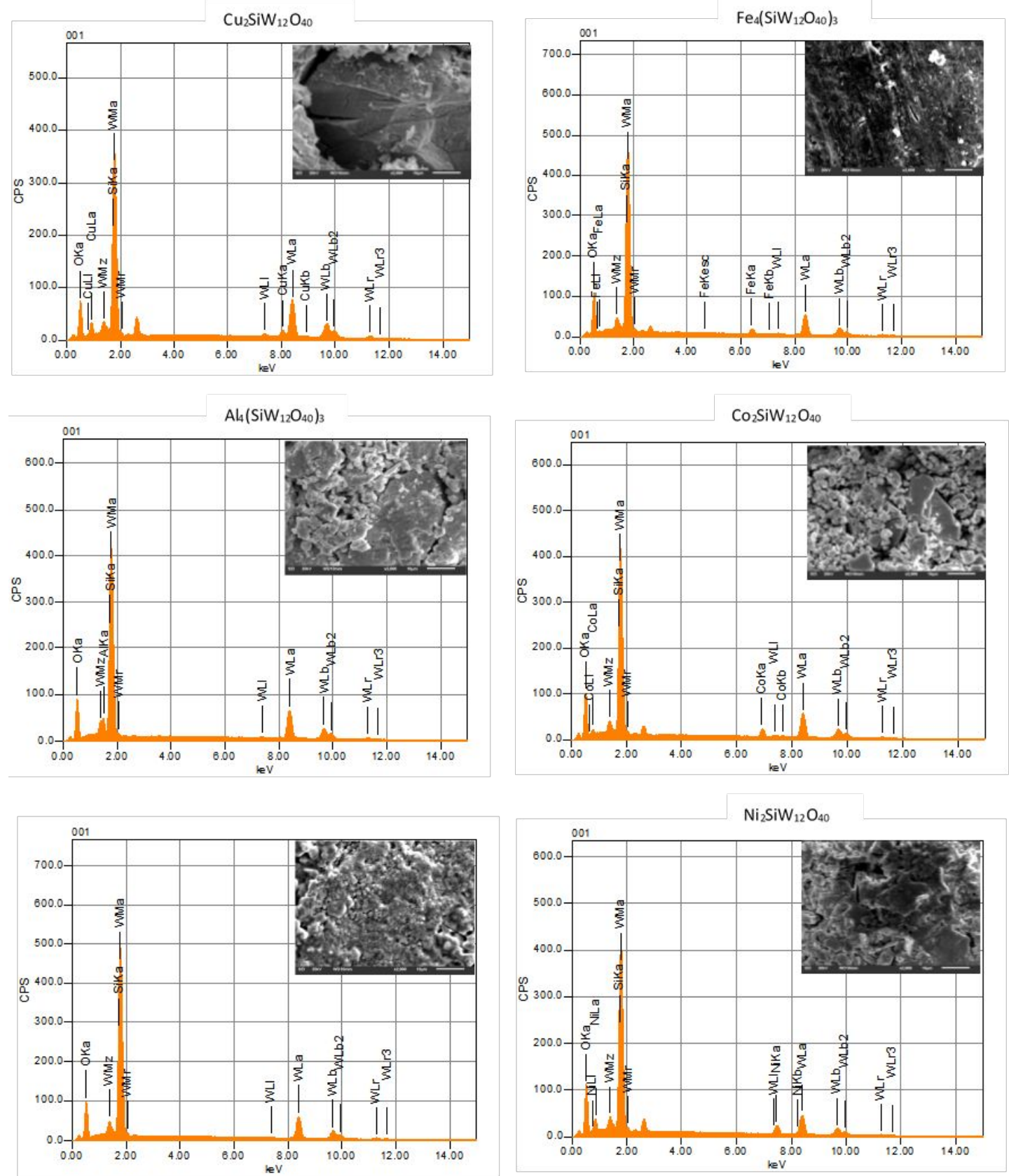

Figure 1SM. EDS spectra and MEV images of the silicotungstic acid and their metal exchanged salts 
Table 1SM. Crystallite size of of silicotungstic acid and their transition metal salts (adapted from ref. 48$)^{\mathrm{a}}$

\begin{tabular}{cc}
\hline Catalyst & $\begin{array}{c}\text { Crystallite size } \\
(\mathrm{nm})^{\mathrm{a}}\end{array}$ \\
\hline $\mathrm{H}_{4} \mathrm{SiW}_{12} \mathrm{O}_{40}$ & 26 \\
$\mathrm{Ni}_{2} \mathrm{SiW}_{12} \mathrm{O}_{40}$ & 31 \\
$\mathrm{Cu}_{2} \mathrm{SiW}_{12} \mathrm{O}_{40}$ & 24 \\
$\mathrm{Co}_{2} \mathrm{SiW}_{12} \mathrm{O}_{40}$ & 36 \\
$\mathrm{Al}_{4 / 3} \mathrm{SiW}_{12} \mathrm{O}_{40}$ & 26 \\
$\mathrm{Fe}_{4 / 3} \mathrm{SiW}_{12} \mathrm{O}_{40}$ & 42
\end{tabular}

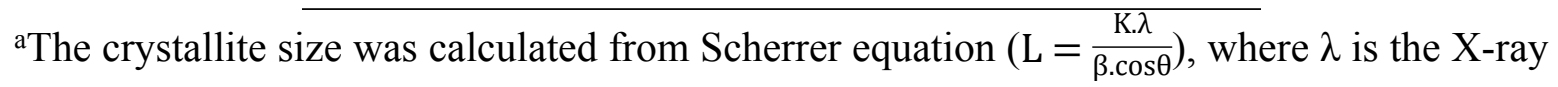
wavelength in nanometer $(\mathrm{nm}), \beta$ is the peak width of the diffraction peak profile at half maximum height resulting from small crystallite size in radians, and $\mathrm{K}$ is a constant related to crystallite shape, normally taken as 0.89 . 
Table 2SM. Water mol/ catalyst mol obtained from TG analyses of the silicotungstic catalysts (adapted from ref. 48)

\begin{tabular}{cc}
\hline Catalyst & water/ catalyst mol \\
\hline $\mathrm{H}_{4} \mathrm{SiW}_{12} \mathrm{O}_{40}$ & 11 \\
$\mathrm{Ni}_{2} \mathrm{SiW}_{12} \mathrm{O}_{40}$ & 7 \\
$\mathrm{Cu}_{2} \mathrm{SiW}_{12} \mathrm{O}_{40}$ & 7 \\
$\mathrm{Co}_{2} \mathrm{SiW}_{12} \mathrm{O}_{40}$ & 10 \\
$\mathrm{Al}_{4 / 3} \mathrm{SiW}_{12} \mathrm{O}_{40}$ & 8 \\
$\mathrm{Fe}_{4 / 3} \mathrm{SiW}_{12} \mathrm{O}_{40}$ & 13 \\
\hline
\end{tabular}


Table 3SM. Surface, volume, and pores size of silicotungstic catalysts (adapted from ref. 48)

\begin{tabular}{ccccc}
\hline Entry & Catalyst & $\begin{array}{c}\text { Surface area } \\
\mathrm{BET}^{\mathrm{a}}\end{array}$ & $\begin{array}{c}\text { Pores volume } \\
\left(\mathrm{m}^{3} / \mathrm{g} \times 10^{-2}\right)\end{array}$ & $\begin{array}{c}\text { Pores size }^{\mathrm{b}} \\
(\mathrm{nm})\end{array}$ \\
\hline 1 & $\left.\mathrm{H}^{2} \mathrm{~g}^{-1}\right)$ & & \\
\hline 2 & $\mathrm{Co}_{2} \mathrm{SiW}_{12} \mathrm{O}_{40}$ & 4 & 0.6 & 3.8 \\
3 & $\mathrm{Cu}_{2} \mathrm{SiW}_{12} \mathrm{O}_{40}$ & 9 & 1.1 & 3.3 \\
4 & $\mathrm{Fe}_{4 / 3} \mathrm{SiW}_{12} \mathrm{O}_{40}$ & 5 & 1.6 & 3,2 \\
5 & $\mathrm{Ni}_{2} \mathrm{SiW}_{12} \mathrm{O}_{40}$ & 8 & 0.8 & 3.6 \\
6 & $\mathrm{Al}_{4 / 3} \mathrm{SiW}_{12} \mathrm{O}_{40}$ & 9 & 0.3 & 3.1 \\
\hline
\end{tabular}

${ }^{\mathrm{a} M u l t i p o i n t ~ B E T ~ m e t h o d ~}$

${ }^{\mathrm{b} D F T}$ method 
